\title{
SCHUR CONVEX FUNCTIONS AND THE BONNESEN STYLE ISOPERIMETRIC INEQUALITIES FOR PLANAR CONVEX POLYGONS
}

\author{
JIBING QI AND WEN WANG
}

\begin{abstract}
In this note, we continue to investigate Bonnesen-type isoperimetric inequalities for planar convex polygons. We shall first establish some analytic isoperimetric inequalities for a special class of Schur convex functions. Subsequently, by using these analytic isoperimetric inequalities, Bonnesen-type isoperimetric inequalities and related inverse inequalities for the planar convex polygons are obtained.
\end{abstract}

Mathematics subject classification (2010): 52A10, 51N20. function

Keywords and phrases: Bonnesen-type isoperimetric inequality, planar convex polygon, Schur convex

\section{REFERENCES}

[1] Y. DAI, W. X. XU, J. Z. ZHOU, Some Bonnesen style inequalities and planar isoperimetric Ddficit upper limit, Proceedings of the fourteenth international workshop on Diff. Geom. 14 (2010), 69-76, available online at https://www.researchgate.net/publication/265655647 _Some_Bonnesen_style_inequalities_and_planar_isoperimetric_deficit_upper_limit.

[2] H. T. KU, M. C. KU AND X. M. ZhANG, Analytic and Geometric Isoperimetric Inequalities, Journal of Geometry, vol. 53 (1995), 100-121. MR 96f:52012, available online at http://link.springer.com/article/10.1007\%2FBF01224044.

[3] H. T. KU, M. C. KU AND X. M. Zhang, Isoperimetric Inequalities on the Surfaces of Constant Curvature, Canadian Journal of Mathematics, 49 (1997), 1162-1187, available online at https://www.researchgate.net/publication/265715424_Isoperimetric_inequalities _on_surfaces_of_constant_curvature.

[4] A. W. Marshall, I. Olkin, Inequalities: Theory of Majorization and Its Applications, New York: Academic Press, 1979.

[5] L. MA, A Bonnesen-style inequality for the planar convex polygon, J. of Math. (PRC), 35 (2015), $154-158$.

[6] D. S. Mitrinović, Analytic Inequalities, Springer-Verlag, Berlin, Heidelberg, New York, 1970, MR 43:448.

[7] R. Osserman, The isoperimetric inequality, Bull. Amer. Math. Soc. 84 (1978), 1182-1238, MR 58:18161.

[8] R. Osserman, Bonnesen-style Isoperimetric Inequalities, Amer. Math. Monthly, 86 (1979) 1-29, MR 80h:52013, available online at http://www. jstor.org/stable/2320297.

[9] S. RABINOwITZ, Some Bonnesen-style triangle inequalities, available online at https://www.researchgate.net/publication/268312979_Some_Bonnesen-style_ triangle_inequalities.

[10] C. N. ZENG, L. MA, J. Z. ZHOU, F. W. ChEN, The Bonnesen isoperimetric inequality in a surface of constant curvature, Sci China Math, 55 (2012), 9, 1913-1919, doi: 10.1007/s11425-012-4405-z, available online at https://www.researchgate.net/publication/257682892_The_Bonnesen -isoperimetric_inequality_in_a_surface_of_constant_curvature.

[11] X. M. ZHANG, Bonnesen-style Inequalities and Pseudo-perimeters for Polygons, Journal of Geometry, 60 (1997), 188-201. 
[12] X. M. ZHANG, A Refinement of the Discrete Wirtinger Inequality, Journal of Mathematical Analysis and Applications, 200(1996)687-697, MR 97h:26012, available online at doi:10.1006/jmaa.1996.0232.

[13] X. M. Zhang, Schur-convex functions and isoperimetric inequalities, Proceedings of the American Mathematical Society, 126 (2) (1998), 461-470, available online at http://mathaware.org/ proc/1998-126-02/S0002-9939-98-04151-3/S0002-9939-98-04151-3.pdf.

[14] A. Wayne Roberts and Dale E. Varberg, Convex Functions, Academic Press, New York, San Francisco, London, 1973, MR 56:1201. 